\title{
Mathematical models in flood management: overview and challenges
}

\author{
D. De Wrachien ${ }^{1}, \mathrm{~S}$. Mambretti ${ }^{2} \&$ A. Sole $^{3}$ \\ ${ }^{1}$ Department of Agricultural Engineering, State University of Milan, Italy \\ ${ }^{2}$ DIIAR, Politecnico di Milano, Italy \\ ${ }^{3}$ DIF A, Università degli Studi della Basilicata, Italy
}

\begin{abstract}
One-third of the annual natural disasters and economic losses, and more than half of the respective victims are flood related. These hazards are likely to become more frequent and more relevant in the future, due to the effects of increase in population, urbanization, land subsidence and the impacts of climate change.

Knowledge and advanced scientific tools play a role of paramount importance in the strain of coping with flooding problems. In this context, flood modelling represents the basis for effective flood mitigation.

The modelling approach aims to provide the best means for assessing and, subsequently, reducing the vulnerability of rural and urban flood prone areas.

By using models, an attempt is made to replace trial and error based strategies, as practised in the past, with more physically-based measures of flood management and control. Mathematical models are the best tools, nowadays available, for the design of efficient flood protection strategies and excellent supporters of decision-makers.

With reference to these issues, the paper provides a review and a general description of the main features of the models currently used in flood management along with the characteristics of the experimental data required for models' calibration.

Moreover, to highlight the effectiveness and the resilience of these tools, some case studies of flood mitigation and hazard assessment are presented.
\end{abstract}

Keywords: flood modelling, flood frequency analysis, deterministic models, flood studies worldwide. 


\section{Introduction}

Floods are among the most damaging of natural hazards, and are likely to become more frequent, more relevant and more damaging in the future due to the effects of increase in population, urbanization, land subsidence and, to a certain extent, the impacts of climate change.

In this context, flood modelling represents the basis for effective flood mitigation. They also are essential for flood risk assessment of both the current situation and feasible future scenarios. The modelling approach aims to provide the best tool for assessing and, subsequently, reducing the vulnerability of rural and high value urban flood prone areas as well as industrial zones.

Within this context, mathematical models are used for the following purposes (van Duivendijk [7]):

- $\quad$ simulation of flood waves in rivers and their floodplains;

- assessment of the effectiveness of certain flood protection measures on extent of flooding and damages;

- evaluation of flood damages;

- design and construction of flood risk maps for zoning purposes;

- $\quad$ analysis of the effects of infrastructure and urban developments, as well as changes in land use, on flood;

- $\quad$ flood forecasting and warning;

- $\quad$ education, to increase communication and public awareness.

\section{Types of models}

Research work on flood dynamics has traditionally specialised in different mathematical models. They can roughly categorized into stochastic and deterministic models.

Stochastic models are based on flood frequency analysis, defined as the means by which flood discharge magnitude is related to the probability of its being equalled or exceeded in any year or to its frequency of recurrence or return period.

Deterministic models are, generally, based on physical properties of elements that feature or influence the phenomenon under investigation, such as the catchment characteristics, the channel geometry, the rainfall-runoff process.

\subsection{Stochastic models}

Estimation of floods corresponding to specified return periods is essential for the design of flood protection measures, assessment of regions at risk of flooding, and the pertinent management of flooded areas.

Due to climatic variability, which drives flood events, stochastic modelling has become widely applied to estimate the magnitude of flood corresponding to a specified risk (Chow et al. [5]; Stedinger et al. [13]). 


\subsubsection{Flood frequency analysis}

Flood frequency analysis was developed, in the first instance, in response to the need for information for the safe and economical design of engineering structures, either for the conveyance of flood flows (bridges, culverts, diversion channels, reservoirs, spillways) or for the protection or mitigation from flooding of land and property (embankments and walls). Moreover, this procedure is currently used for planning purposes and the design of land-use categories based on flood zoning with respect to vulnerability.

Frequency analysis is most commonly applied to peak discharges, instantaneous or averaged over a specified duration. Analysis is carried out on an observed historic record of river flow with the aim of assessing future probabilities of exceedence. It is also usually assumed that there will be not temporal change in the underlying statistics due to climate variability or to land use changes.

A large number of probability distributions and methods of application have been used for interpolation or extrapolation.

The two main approaches to select the flood series for fitting a stochastic model to the observed floods are based on the series for maxima annual flows (MAF) or partial duration series of floods (PDF). The MAF series selects the maximum flood event for each year, while the PDF series consists of all flood peaks above a specified magnitude.

The MAF approach ignores the fact that the highest flows in some years can be lower than flood events in other years, and hence it excludes significant high flood events in the parameter estimation process. The PDF procedure, on the other hand, considers all significant flood events in its parameter estimation process and its size can extend far beyond the available years of recorded flows.

Recently, a new relation between the return period of the PDF and the MAF series was proposed (Mohssen [1]), based on the assumption that flood events are independent. The General Extreme Value (GEV) distributions, in addition to the Generalized Pareto (GP) distribution have been fitted and applied to analyse flood events.

\subsection{Deterministic models}

Deterministic modelling (flood routing) can be defined as a mathematical procedure for predicting the changing magnitude, speed and shape of a flow wave as a function of time (i.e. the flow hydrograph) at one or more points along a watercourse. The watercourse may be a river, stream, reservoir, estuary, canal, discharge ditch, or storm sewer. The flow hydrograph can result from precipitation runoff, reservoir releases, landslides into reservoirs, or tides. Flood routing may be classified as hydrologic (lumped), or hydraulic (distributed), or hybrid.

\subsubsection{Hydrologic models}

Hydrologic modelling involves the balancing of inflow, outflow and volume of storage through the use of continuity equation. A second relationship, the storage-discharge relation, is also required between outflows rate and storage in 
the system. This implies that water surface is level through the watercourse, usually a reservoir or lake. More complex relationships are to be sought for long and narrow reservoirs or open channels where storage is a function of both inflow and outflow. Both graphical and mathematical techniques for solving the continuity equation have been proposed.

The attractiveness of hydrologic modelling consists in its relative simplicity compared with hydraulic models. However, these procedures neglect backwater effects and are not accurate for rapidly rising hydrographs routed through mild to flat sloping rivers. They are also inaccurate for rapidly rising hydrographs in long reservoirs.

Hydrologic models can be categorized as (Fread [8]):

- level-pool types, used for reservoirs;

- $\quad$ storage types, used for rivers;

- linear systems types, which assume that the routing channel is composed of linear reservoirs connected by linear channels.

\subsubsection{Hydraulic models}

A good understanding of a complex flooding event can only be achieved by means of hydraulic models, because the flow rate, velocity, and depth vary in space along the watercourse and across a floodplain and/or a flood prone area. Estimation of these properties can be obtained by using the complete differential equations of 1D or 2D unsteady flow, known as the De Saint Venant (SV) or shallow water (SW) equations. Those equations allow the flow rate and water level to be computed as functions of space and time rather than time alone, as in the lumped flow routing methods. Distributed flow routing based on the complete SV or SW equations is known as hydrodynamic routing.

In some cases, the governing equations can be simplified to a $1 \mathrm{D}$ continuity equations and a uniform flow relationship, referred to as kinematic wave routing, which implies that the discharge can be computed as a simple function of depth alone. Uniform flow implies a balance between gravitational and frictional forces in the channel. This assumption can rarely be justified, especially on very flat slopes where effects of water surface cannot be ignored.

Cases where other terms in the momentum equation for hydraulic routing must be taken into account include:

- upstream movement of tide or storm surges;

- backwater effects from downstream reservoirs and tributary inflows;

- flood waves in channels of very flat slope;

- abrupt waves caused by sudden releases from reservoirs or dam failures.

Selection of a flow routing model for a particular application is influenced by the relative importance placed on the following factors:

- the model's suitability to answer the user's questions;

- the model's accuracy;

- the type and availability of required data;

- the complexity of the mathematical formulation.

Distributed flow routing models are useful tools for determining floodplain depths, required heights of structures (bridges or levees), inundation maps for 
dam-break contingency planning, transient waves created in reservoirs by gate or turbine changes, landslide-produced waves in reservoirs and unsteady flow in storm sewer systems. The real flow process in each of these applications varies in all three space dimensions. However, normally the spatial variation of the flow characteristics can be approximated as varying in only one space dimension - the direction along the flow channel. Thus the 1D equations of unsteady flow are widely used.

\subsubsection{Hybrid models}

Until recently, hydraulic models were not reckoned a practical alternative for flood routing because they were considered not economically viable to obtain cross section data over the reaches involved in flood routing.

Recent investigations (Hicks [9]) have revealed that hydraulic routing can be successfully used to determine discharge hydrographs in reaches where little channel geometry data are available, by approximating the model reach by a rectangular channel.

It was found that this "limited geometry" modelling approach - based on 1D SV equations - could accurately determine discharge hydrographs, making it an effective and suitable alternative to hydrologic flood routing. It was also found that this hybrid model offers the advantage of operationally combining the flood routing and the determination of flood levels (Blackburn and Hicks [4]). In addition, the use of a hydraulic model opens up the potential for modelling more dynamic flood events such as ice jam release surges, which cannot be handled by traditional hydrological modelling approaches.

In practical applications, flood forecasting involves two steps.

First, a flood routing model (usually hydrologic) is used to obtain the flood peak by routing flood events between streamflow gauging stations. This flood wave must then be put into a hydraulic model based on detailed channel geometry in order to forecast flood events at key-sites.

A new deterministic approach uses unsteady flow hydraulic modelling for both flood routing and flood level determination. This hybrid model offers the advantage of operationally combining the flood routing and the determination of the flood level. Moreover, this procedure opens up the potential for modelling more dynamic flood events such as ice jam release surges, which cannot be handled by traditional hydrologic or hydraulic modelling approaches.

\section{Data: requirements and problems}

It is a demanding task to precisely define the input data needed for flood modelling. The great variety of models and the different purposes for which they are designed and proposed make it difficult to devise general rules feasible for all situations.

Within this context, the most important question to be addressed when defining the quantity and quality of data to be used as input of a model is the purpose pursued. Modelling a flooding event in a specific area, caused by the failure of a dam, and over a wide rural plain due to heavy rain, concerns different 
data and ask for different approaches. In the event of dam failure, precise information on topography and soil characteristics are much more necessary than data on earlier flooding, while in flood forecasting over a broad area, data of historic flooding are essential.

Hydrologic models, based on empirical storage-flow relations to approximate momentum effects, require only streamflow hydrographs as inputs. Hydraulic models require additional, physical and altimetric data describing the channel geometry and the floodplain morphology, in order to forecast flood levels at keysites.

To this end, topographic data, referring to longitudinal and cross sections of the river through its banks and dykes or outlining flood zones play a role of paramount importance. Longitudinal and cross sections alone do not suffice for investigations involving 2D models, which require the ground to be divided into cells interrelated by means of weirs, culverts, or other hydraulic structures.

The topographic data resolution strongly affects the flood model efficiency. Sound Digital Elevation Models (DEMs) must provide an accurate description of micro-topography (e.g. levees, embankments, roads, buildings) to create a computational mesh in which all the elements that affect flow dynamics and flood propagations are included. Advances in models and in remote sensing techniques make possible to generate high resolution DEMs for whole watersheds. The latest developments in airborne laser scanning, makes it feasible to produce high quality digital surface models (DSMs) with accuracies less than $\pm 25 \mathrm{~cm}$ depending on the land cover, slope, flight parameters and environmental conditions (Sole et al. [12]).

Hydrological/hydraulic flow characteristics are fundamental in flood modelling along with boundary conditions on depths and discharges.

Either a specified discharge or water-surface time series can be used for both upstream and downstream flow conditions. The downstream boundary condition can also be a critical flow section such as the entrance to a waterfall or a steep reach.

The resistance to flow in a watercourse may be parameterized by the Manning, Chézy, Darcy friction coefficients which represent the effect of roughness elements of the channel bed and particles as well as losses mainly due to dynamic bed morphology and vegetation. Best results are obtained when the friction coefficients are adjusted (calibrated) to reproduce historical observation of stage and discharge.

A good understanding of flooding event can only be achieved by means of 1D or $2 \mathrm{D}$ hydrodynamic models. These models have to simulate the movement of a relatively shallow layer of water (1-4 $m$ deep) over a vast, nearly flat area. As soon as the depth of the water layer drops down to $0.2-0.5 \mathrm{~m}$ it becomes difficult to simulate the flow. The reason lies in the 4D behaviour of this phenomenon in large, nearly inaccessible (during flooding) environments, in which at any point the direction, the velocity and the depth of the current vary as a function of location, ground level and time. As a matter of fact, the flood wave moves through a floodplain in the course of a few days and during this short period of 
time it is very difficult to make the observations (water level, direction and speed of current) required for the calibration of complex unsteady flow models.

\section{Case studies}

To highlight the effectiveness and the resilience of the hydrodynamic simulation models, three case studies of flood mitigation and hazard assessment are featured. The first deals with laboratory measurements reproducing dam-break waves with floating debris, the last two concern river basins in Italy and Canada. After describing the methodologies, which differ in both the effort required and the accuracy expected, the chapter focuses on both the results achieved and the reliability of the procedures applied.

\subsection{Calibration of dam-break waves (Laboratory tests)}

Flash floods triggered by a sudden collapse of a dam (dam-break) are often characterized by the formation on shock waves with floating debris caused by many factors, such as valley contractions, irregular bed slopes and non-zero tail water depth. It is commonly accepted that a mathematical description of these phenomena can be accomplished by means of 1D or 2D SV equations (Bellos and Sakkas [3]; Bechteler et al. [2]; Aureli et al. [1]).

To validate the model, comparisons have been made between its predictions and experimental results carried out in the Hydraulic Laboratory of the Politecnico di Milano. The tests were performed with flows of water and

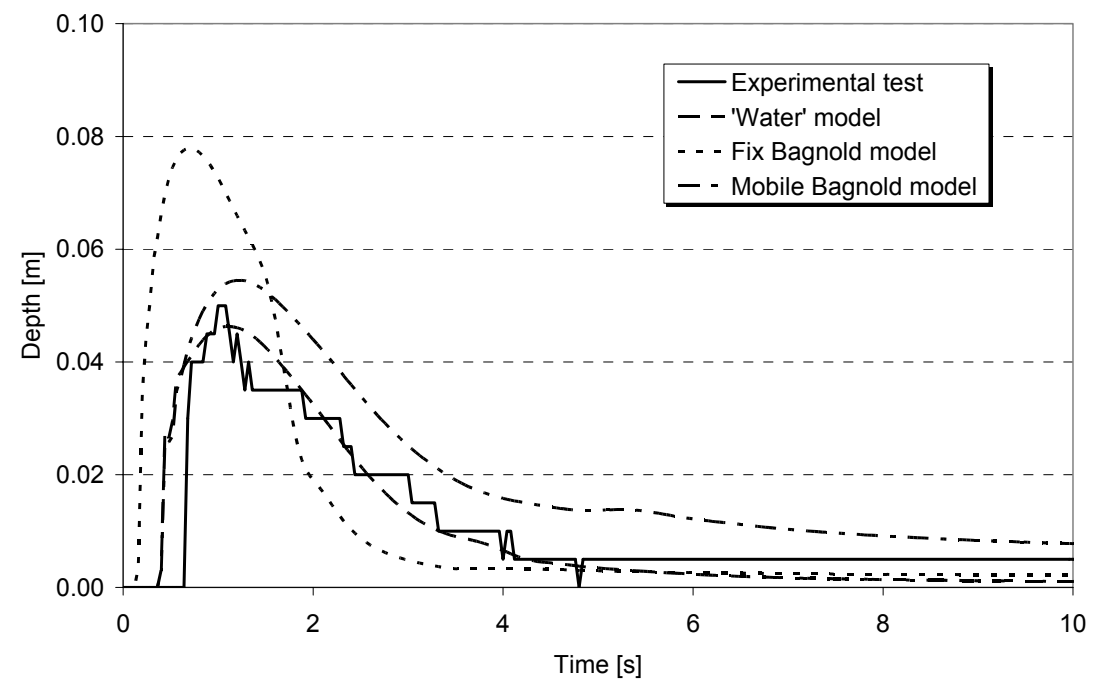

Figure 1: Debris flow wave in some characteristic sections of the experimental channel. Comparison between mathematical model and experimental results. Water-gravel, abs 200, conc. $40 \%$, slope $15^{\circ}$, smooth bottom. 


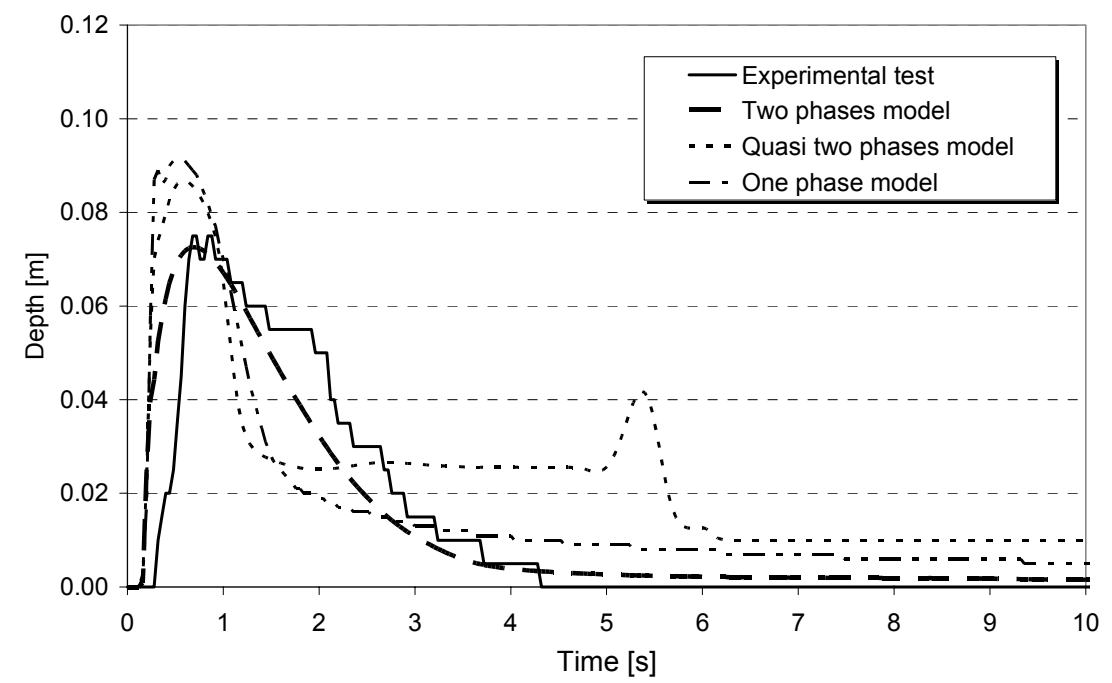

Figure 2: Debris flow wave in some characteristic sections of the experimental channel. Comparison between mathematical model and experimental results. Water-gravel, abs 140 , conc. $40 \%$, slope $20^{\circ}$, smooth bottom.

mixtures in a uniform geometry flume reproducing dam-break waves (Larcan et al. [10]; De Wrachien and Mambretti [6]).

To take into account different behaviours of the flow, the experimental data have been compared with the predictions of three rheological laws included in the one phase model (called "Water", "Fix Bagnold" and "Mobile Bagnold") and with those of the two phase model.

Comparisons show good agreement on the general shape that includes a steep front immediately followed by the maximum wave height and a decrease in flow depth down to an asymptotic value reached at the stoppage (figure 1 and 2).

The model can easily be extended to channels with arbitrary cross sections for both mature (non-stratified) and immature (stratified) debris flow routing, as well as for solving different problems of unsteady flow in open channels by incorporating the appropriate initial and boundary conditions.

\subsection{The Basento case study}

This study is included in a larger investigation that comprises all rivers of the Basilicata Region. The Basento River is $157 \mathrm{~km}$ long, with a river basin of 1535 $\mathrm{km} 2$. The aim was to assess the flooding risk for the whole region, in order to comply with the Italian land protection legislation, defined by the regional Authority by means of the Hydro Geologic Safety Plan (PAI).

This plan aims to identify the different risk zones and to devise and work out flooding area maps for flow rates corresponding to different return periods. 
Four codes have been used for the simulation: Hec Ras, Mike 11 and Mike 21 (Danish Hydraulic Institute) and FLO-2D in order to compare the effectiveness of the different tools and to identify the most appropriate one.

The 1D simulations have been carried out using field data of more than 650 river cross sections (including bridges, culverts, weirs and other hydraulic works) in order to assess both the geometric characteristics of streams and peak flows with 30, 200 and 500 years return period, respectively, and to use as upstream boundary conditions for the $2 \mathrm{D}$ simulations.

It is worth underline that integration between hydrodynamic model and detailed field data (as laser scanning data) is quite difficult and requires a great deal of pre-processing work. To this end, values of both water-surface elevation and top width in river bed, obtained within the simulation process, have been used in the GIS procedures, to draw up maps of different flooded areas.

The differences between the simulations of the two 1D models reflect the differences between the solvers of the model's basic equations. Anyhow, these differences are meaningless, taking into account the scale of the maps (1:5000 scale, $5 m$ contour line).

The area investigated covers a strip of about $3.5 \mathrm{~km}$ on both the left and right sides of the river. The set of topographic data, obtained by means of laser scanning was used to generate the Digital Surface Model (DSM), which allowed to design a Key Points Model (KPM) - containing data of paramount importance for topographic maps - and depict the bathymetry of the investigated area.
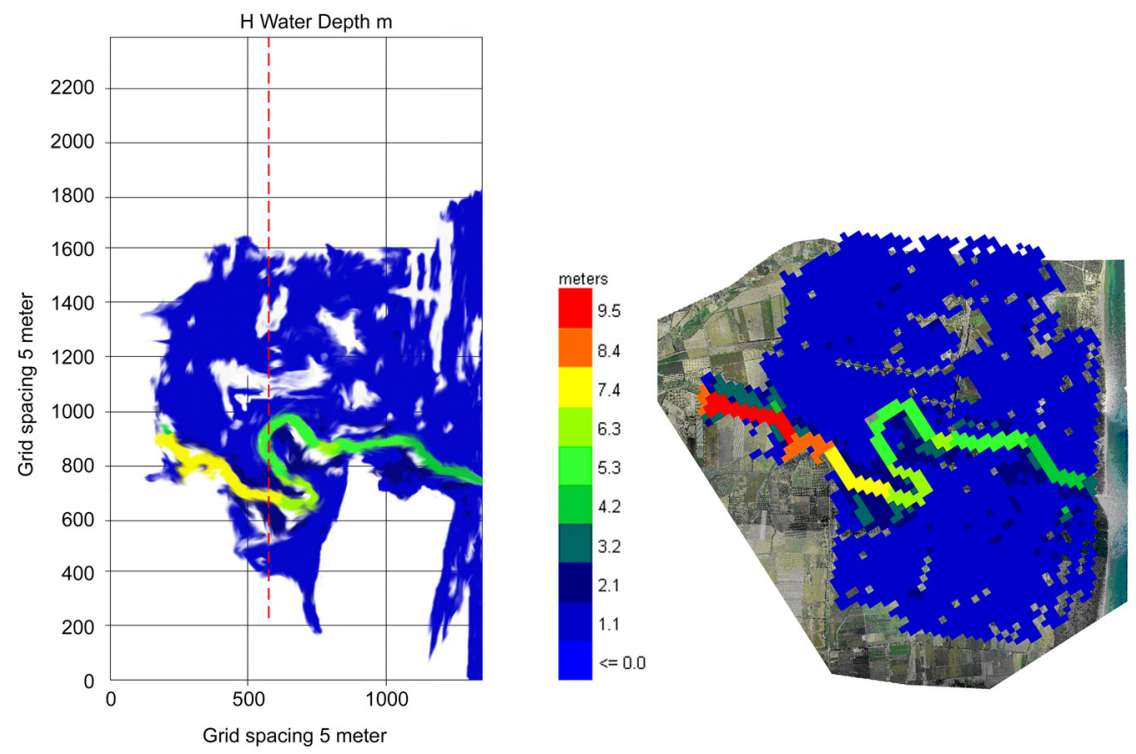

Figure 3: Basento River: inundation maps for flood with 30 years return period, (left) Mike 21 and (right) FLO-2D results. 
With regard to the $2 \mathrm{D}$ models, figure 3 shows the differences between two flooded areas (return period equal to 30 years) obtained by means of Mike 21 and FLO-2D models.

\subsection{The Peace River case study}

The objective of this study was to determine whether it is possible to combine flood forecasting and flood level determination using an hybrid model (Blackburn and Hicks [4]). For this investigation, the 1987 summer flood event on the Peace River, Alberta, was routed over an approximately $800 \mathrm{~km}$ reach using a rectangular channel approximation, except through the towns where natural channel geometry was used.

The hybrid model was based on two formulations of the 1D unsteady open channel flow SV equations, which were both solved using the characteristic dissipative Galerkin finite element scheme (cdg1D model). In the routing reaches between towns the cdg1D model, based on a rectangular channel equation formulation, was applied. For the flood level forecasting, the model was modified to incorporate natural channel characteristics. The revised model was named cdg1Dn model. To calibrate the models, the Water Survey of Canada (WSC) water level data, available at a few natural channel subreaches, were used (figure 4).
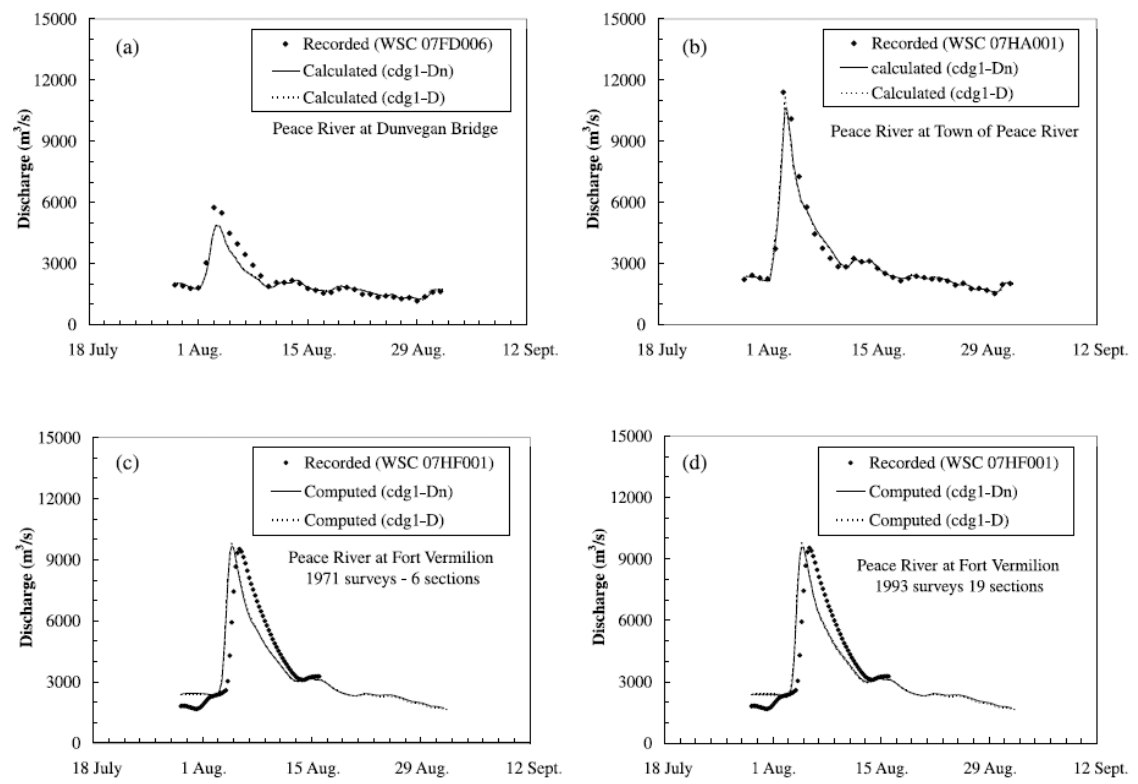

Figure 4: $\quad$ Comparisons of measured and computed water levels for the 1987 flood event: (a) Peace River at Dunvegan Bridge; (b) Peace River at town of Peace River; (c) Peace River at Fort Vermilion; (d) Peace River at Fort Vermilion. 
The results of this case study indicate that flood forecasting and flood level determination can be combined operationally using hybrid flood routing techniques. Moreover, the outcomes pointed out that channel roughness is the primary factor affecting the accuracy of the peak discharge, while channel gradient is critical to obtaining the correct timing for peak arrival.

\section{Concluding remarks}

Floods are among the most damaging of natural hazards, and are likely to become more frequent, more relevant and more damaging in the future due to the effects of increase in population, urbanization, land subsidence and, to certain extent, the impact of climatic change.

A flood event is a natural event of great complexity. The hydrological parameters of a flood (magnitude, frequency, celerity, volume, duration) reflect the stochastic behaviour of precipitation, interception, infiltration, evapotraspiration, soil moisture, overland and ground flows and river channel hydraulics. Models not only help in understanding these flood phenomena, but are also essential for flood risk assessment of the current situation and for assessment of expected changes.

By using models an attempt is made to replace trial and error-based strategies as practised in the past with more physically-based measures. The modelling tool aims to provide the best concept for assessing and reducing the vulnerability of rural and high-value urban flood-prone areas as well as industrial zones.

Within this context, the paper provides a review and a general description of the main features and characteristics of the mathematical models currently used in flood management, mitigation and control, along with the quantity and quality of experimental data to be used both as input and for model calibration.

Mathematical models are useful tools for the design of efficient flood protection strategies and excellent supporters of decision makers, since they enable a complex evaluation of cost benefit analysis of particular proposal for flood protection measures.

\section{References}

[1] Aureli F., Mignosa P., Tomirotti M. "Numerical simulation and experimental verification of dam-break flow with shocks" Journal of Hydraulic Research, 38, pp. 197-216, 2000

[2] Bechteler W., Kulish H., Nujic M. "2D dam-break flooding wave: comparison between experimental and calculated results" Floods and Flood Management, Ed. Saul, Dodrecht, 1992

[3] Bellos V., Sakkas J.G. "1D dam-break flood propagation on dry bed" Journal of Hydraulic Engineering, ASCE 113(12), pp. 1510-1524, 1987

[4] Blackburn J., Hicks F.E. "Combined flood routing and flood level forecasting" Canadian Journal of Civil Engineering, Vol. 29, pp. 64-75, 2002 
[5] Chow V.T., Maidment D.R., Mays L.W. Applied Hydrology McGraw Hill, New York, 1988

[6] De Wrachien D., Mambretti S. "Dam-break shock waves: a two-phase model for mature and immature debris flow" Proc. of the $2^{\text {nd }}$ Int. Conf. on Monitoring, Simulation, Prevention and Remediation of Dense and Debris Flow, De Wrachien D., Lenzi M., Brebbia C. (eds), 18-20 June 2008

[7] Duivendijk J. van Manual on Planning of Structural Approaches to Flood Management. ICID Guideline, New Delhi, India, 2005

[8] Fread D.L. "Flow Routing", Chapter 10, Handbook of Hydrology Maidment D.A. (Ed), McGraw Hill, New York, 1992

[9] Hicks F.E. "Hydraulic flood routing with minimal channel data: Peace River, Canada" Canadian Journal of Civil Engineering, Vol. 23, pp. 524535,1996

[10] Larcan E., Mambretti S., Pulecchi M., "A procedure for the evaluation of debris flow stratification." Proceedings of the $1^{\text {st }}$ International Conference on Monitoring, Simulation, Prevention and Remediation of Dense and Debris flow Lorenzini, Brebbia and Emmauouloudis (eds), Rhodes, Greece, 7-9 June 2006

[11] Mohssen M. "An insight into flood frequency for design floods." Proc. of the $1^{\text {st }}$ International Conference on Flood Recovery Innovation and Response, WIT Press, London, 2008

[12] Sole A., Giosa L., Nolè L., Medina V., Bateman A. Flood risk modelling with LiDAR technology Proc. of the $1^{\text {st }}$ International Conference on Flood Recovery Innovation and Response, WIT Press, London, 2008

[13] Stedinger J.R, Vogel R.M., Foufoula-Georgiou E. "Frequency Analysis of Extreme Events." Chapter 18, Handbook of Hydrology Maidment D.A. (Ed), McGraw Hill, New York, 1992 